\title{
THE NORTH-SOUTH ASYMMETRY OF SOFT X- RAY FLARE INDEX DURING SOLAR CYCLES 21, 22 AND 23
}

\author{
BHUWAN JOSHI and ANITA JOSHI \\ State Observatory, Naini Tal, 263 129, India (E-mail: bhuwan@upso.ernet.in, \\ anita@upso.ernet.in)
}

\begin{abstract}
In this paper the $\mathrm{N}-\mathrm{S}$ asymmetry of the soft X-ray flare index $\left(F I_{S X R}\right)$ during the solar cycles 21, 22 and 23 has been analyzed. The results show the existence of a real $\mathrm{N}-\mathrm{S}$ asymmetry which is strengthened during solar minimum. The slope of the regression lines fitted to the daily values of asymmetry time series has been found to be negative in all the three cycles. The yearly asymmetry curve can be fitted by a sinusoidal function with a period of eleven years. The power spectral analysis of daily asymmetry time series reveals the significant periods of around 28.26 days, 550.73 days and 3.72 years.
\end{abstract}

\section{Introduction}

It is well known that many types of solar phenomena exhibit some form of north-south (N-S) asymmetry. Bell (1962) found a long term asymmetry in the sunspot area data for cycle 8 through 18. Roy (1977) studied the N-S distribution of major solar flares from 1955 to 1974, covering cycles 19 and 20, and found an asymmetry in favour of northern hemisphere which is more pronounced during the minimum of solar cycle. Knoška (1985) studied the asymmetry of flare activity during the years $1937-1978$ using the $\mathrm{H} \alpha$ flare index $\left(F I_{H \alpha}\right)$ introduced by Kleczek $(1952,1953)$ and found no unique relationship between the asymmetry of flare activity and eleven year solar cycle. Vizoso and Ballester (1987) have studied the sudden disappearance (SD) of solar prominence during cycles $18-21$ and found that the N-S asymmetry curve is not in phase with the solar cycle and peaks at or around the solar minimum and that the asymmetry changes its sign during the maximum, at the time of reversal of the Sun's general magnetic field. Verma (1993) reported a study of various solar phenomena occurring in both northern and southern hemisphere of the Sun for solar cycles 8-22. Joshi (1995); Li, Schimider, and Li (1998) and Ataç and Özgüç (1998) studied the N-S asymmetry during the solar cycle 22 with different manifestations of solar activity. In all these studies the asymmetry during this cycle was found to be in favour of southern hemisphere. Ataç and Özgüç (2001) studied the N-S asymmetry of

(C) 2021 Kluwer Academic Publishers. Printed in the Netherlands. 
$F I_{H \alpha}$ during the rising phase of solar cycle 23 (1996-1999) and found that the activity prefers the northern hemisphere in general during the present cycle. Recently Li et al. (2002) have presented the dominant hemisphere of solar activity in each of solar cycle 12 to 22 by calculating the actual probability of the hemisphere distribution of sunspot groups, sunspot areas, the relative number of sunspots, flare index and number of SDs.

Studies of periodicity in time series of N-S asymmetry data have also been made by several authors. Vizoso and Ballester (1989) found a significant peak of around 3.1-3.2 years in the power spectrum performed with the values of flare number and flare index N-S asymmetry. Carbonell, Oliver, and Ballester (1993) and Oliver and Ballester (1994) studied the periodicity in the N-S asymmetry of sunspot area from 1874-1989 and 1874-1993 respectively. They obtained statistically significant period of 163.93 rotations (12.1 years). Özgüç and Ataç (1996) performed the power spectral analysis of $\mathrm{N}-\mathrm{S}$ asymmetry time series of flare index values of solar cycle 22 and confirmed the 25.5 day fundamental period of the sun which was discovered by Bai and Sturrok (1991). Taking all this into account, here we have made an attempt to investigate the $\mathrm{N}-\mathrm{S}$ asymmetry of soft X-ray flare index $\left(F I_{S X R}\right)$, which is based on the continuous record of SXR flares observed by GOES during solar cycles 21,22 and 23 . The daily values of $F I_{S X R}$ include the contribution of SXR flares of different classes (B to X) and are suitable for a short and intermediate term solar activity analysis. In the paper we have also searched for the periodicity in the daily asymmetry time series of $F I_{S X R}$ for the period 1975-2003.

\section{The Soft X-ray Flare Index}

For the present analysis, first we have calculated daily $F I_{S X R}$ by using the data of SXR flares observed by GOES in $1-8 \AA$ wavelength band. The data for the time span of 01 September 1975 to 30 June 2003 has been downloaded from NGDC's anonymous ftp server: ftp://ftp.ngdc.no aa.gov/STP/SOLAR_DATA/SOLAR_FLARES/XRAY_FLARES. During this period the occurrence of 56,072 SXR flares are reported.

The $F I_{S X R}$ is introduced by Antalová (1996) by weighing the SXR flares of classes $\mathrm{C}, \mathrm{M}$ and $\mathrm{X}$ as 1,10 and 100 respectively (in units of $10^{-6} \mathrm{Wm}^{-2}$ ). In this manner, for example, the SXR flare index for individual flares of class C7.3, M2.9 and X4.8 is 7.3, 29.0 and 480 respectively (See also Landi et al., 1998). Here, we have also included the contribution of flares of class B in the calculation of daily $F I_{S X R}$. Suppose $m_{B}, m_{C}, m_{M}$ and $m_{X}$ are the digit multipliers for the flares 


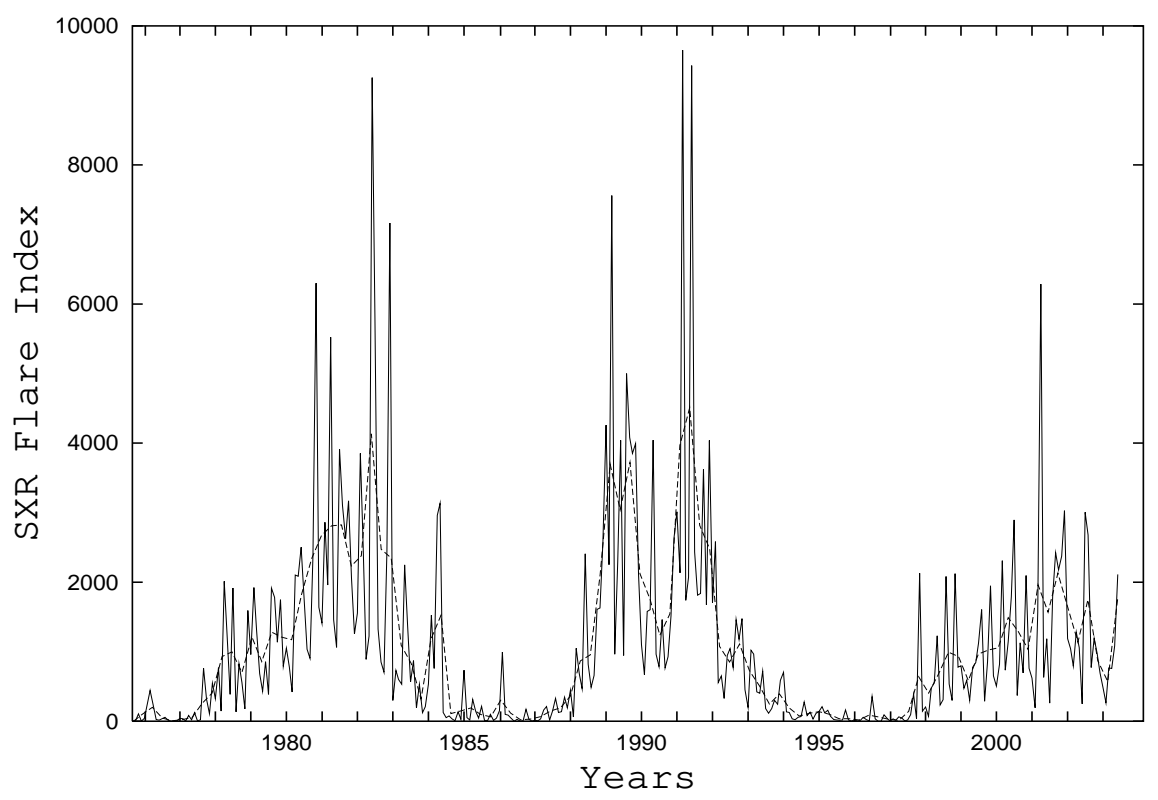

Figure 1. Plot of the monthly values of $F I_{S X R}$ for the period 1975-2003. Dashed line shows the smooth spline curve.

of class $\mathrm{B}, \mathrm{C}, \mathrm{M}$ and $\mathrm{X}$ respectively, then the expression to calculate daily $F I_{S X R}$ can be written in the following way

$F I_{S X R}=0.1 \times \sum_{i} m_{B}+1.0 \times \sum_{j} m_{C}+10.0 \times \sum_{k} m_{M}+100.0 \times \sum_{l} m_{X}$

where $i, j, k$ and $l$ are the number of flares of class $\mathrm{B}, \mathrm{C}, \mathrm{M}$ and $\mathrm{X}$ per day respectively.

Figure 1 shows the variation of monthly values of $F I_{S X R}$ for the period of 1975-2003 covering solar cycles 21, 22 and the rising and maximum phase of cycle 23 . We have compared $F I_{S X R}$ with $F I_{H \alpha}$. For this a correlation plot of these activity indicators is given in Figure 2. The $F I_{H \alpha}$ values are available for general use in Kandilli observatory's and NGDC's anonymous ftp servers: ftp://ftp.koeri.boun.edu.tr/pub/astronomy/flare_index and ftp://ftp.ngdc.noaa.gov/STP/SOLAR_DATA/ SOLAR_FLARES/INDEX. 


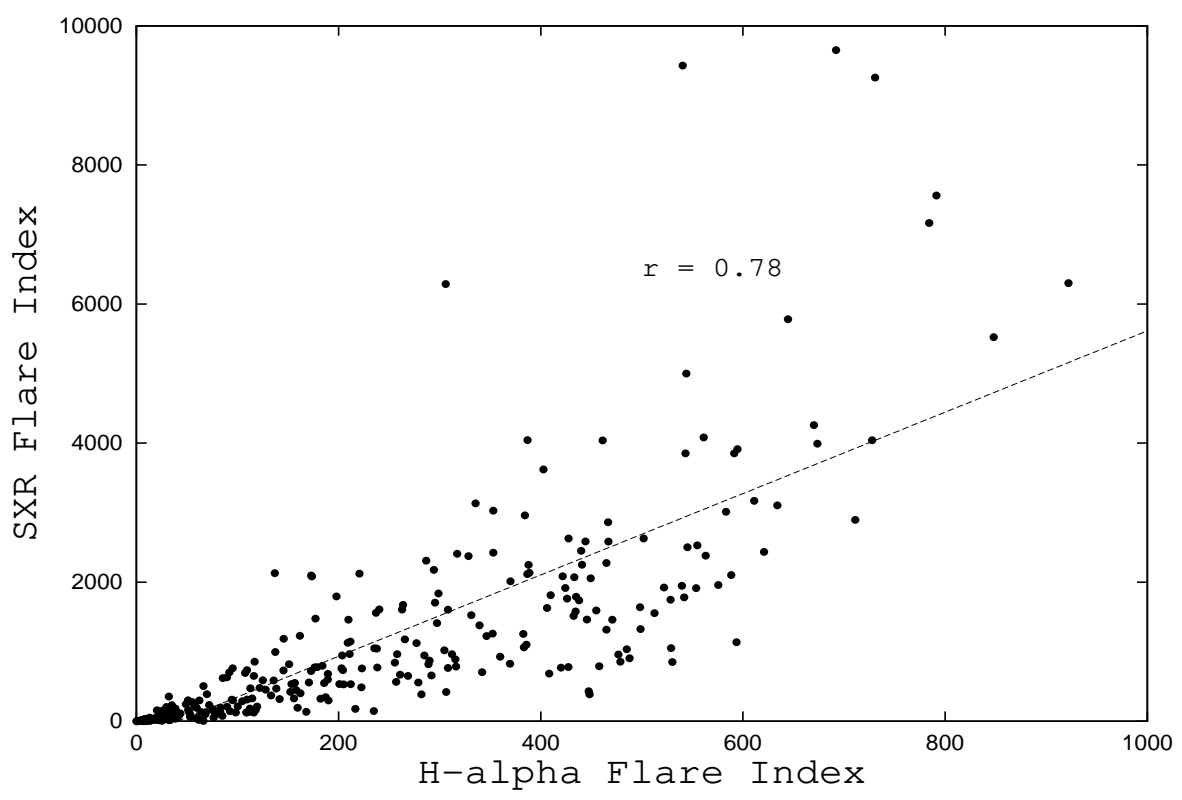

Figure 2. Scatter diagram showing the correlation between the monthly values of $F I_{S X R}$ and $F I_{H \alpha}$. Here $\mathrm{r}$ indicates the correlation coefficient.

\section{The Analysis of Asymmetry Time Series}

The N-S asymmetry for the $F I_{S X R}$ is defined as

$$
A_{S X R}=\frac{F I_{N}-F I_{S}}{F I_{N}+F I_{S}}
$$

where $F I_{N}$ and $F I_{S}$ stands for the daily $F I_{S X R}$ in the northern and southern solar hemisphere respectively. Thus, if $A_{S X R}>0$, the activity in the northern hemisphere dominates and if $A_{S X R}<0$, the reverse is true. In the GOES flare list the heliographic latitudes of all the flares are not known and SXR flares of class $\geq$ B 1.0 are not occurred daily. Therefore such data were excluded from our study. Due to these reasons the above expression gives us an asymmetry time series composed of 6952 values. In Figure 3 the plot of daily asymmetry time series is presented. To show the reality of the variations of asymmetry time series we have fitted a straight line to the daily values of $A_{S X R}$ for each cycle separately.

To investigate to what extent the asymmetry is real we have followed the method of Letfus (1960) in which we can define the asymmetry of 


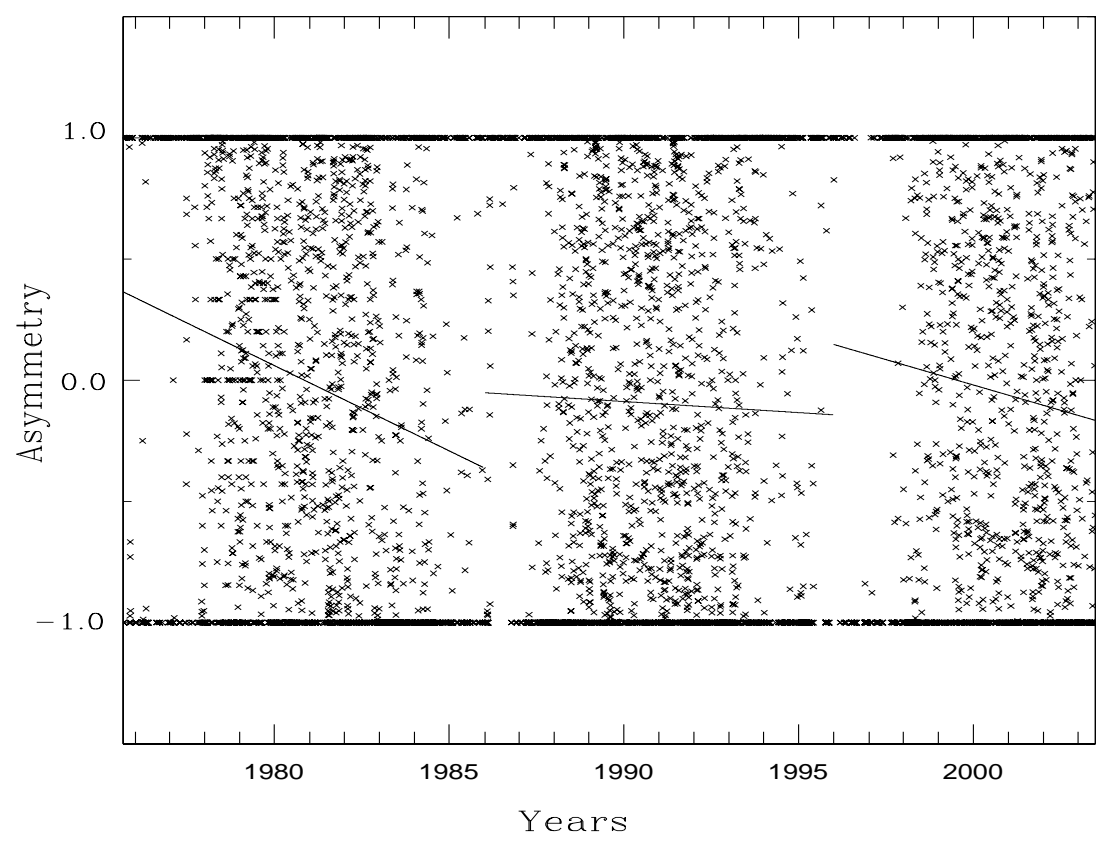

Figure 3. The plot of the daily values of asymmetry time series during solar cycles 21, 22 and 23. The straight line corresponds to the fit of first order polynomial.

random distribution on the solar disk as

$$
\Delta A_{S X R}= \pm \frac{1}{\sqrt{2\left(F I_{N}+F I_{S}\right)}}
$$

which depends upon the values of $F I_{S X R}$ in the northern and southern hemisphere respectively. To verify the reliability of calculated asymmetry values, $\chi^{2}$ test is applied with

$$
\chi=\frac{2\left(F I_{N}-F I_{S}\right)}{\sqrt{2\left(F I_{N}+F I_{S}\right)}}=\frac{\sqrt{2} A_{S X R}}{\Delta A_{S X R}} .
$$

Thus for $A_{S X R}<\Delta A_{S X R}, \Delta A_{S X R} \leq A_{S X R}<2 \Delta A_{S X R}$ and $A_{S X R} \geq$ $2 \Delta A_{S X R}$, the probability that $\mathrm{N}-\mathrm{S}$ asymmetry exceeds the dispersion value is $\mathrm{p}<84 \%, 84 \% \leq \mathrm{p}<99.5 \%$ and $\mathrm{p} \geq 99.5 \%$ respectively. Here the first, second and third limits imply for the statistically insignificant, significant and highly significant values respectively. Using the method of Letfus we have found that out of total 6952 cases, $\mathrm{p} \geq 99.5 \%$ in 4789 cases; $84 \% \leq \mathrm{p}<99.5 \%$ in 1237 cases and $\mathrm{p}<84 \%$ in 926 cases, which means that, in general the $\mathrm{N}-\mathrm{S}$ asymmetry is a real phenomenon and 


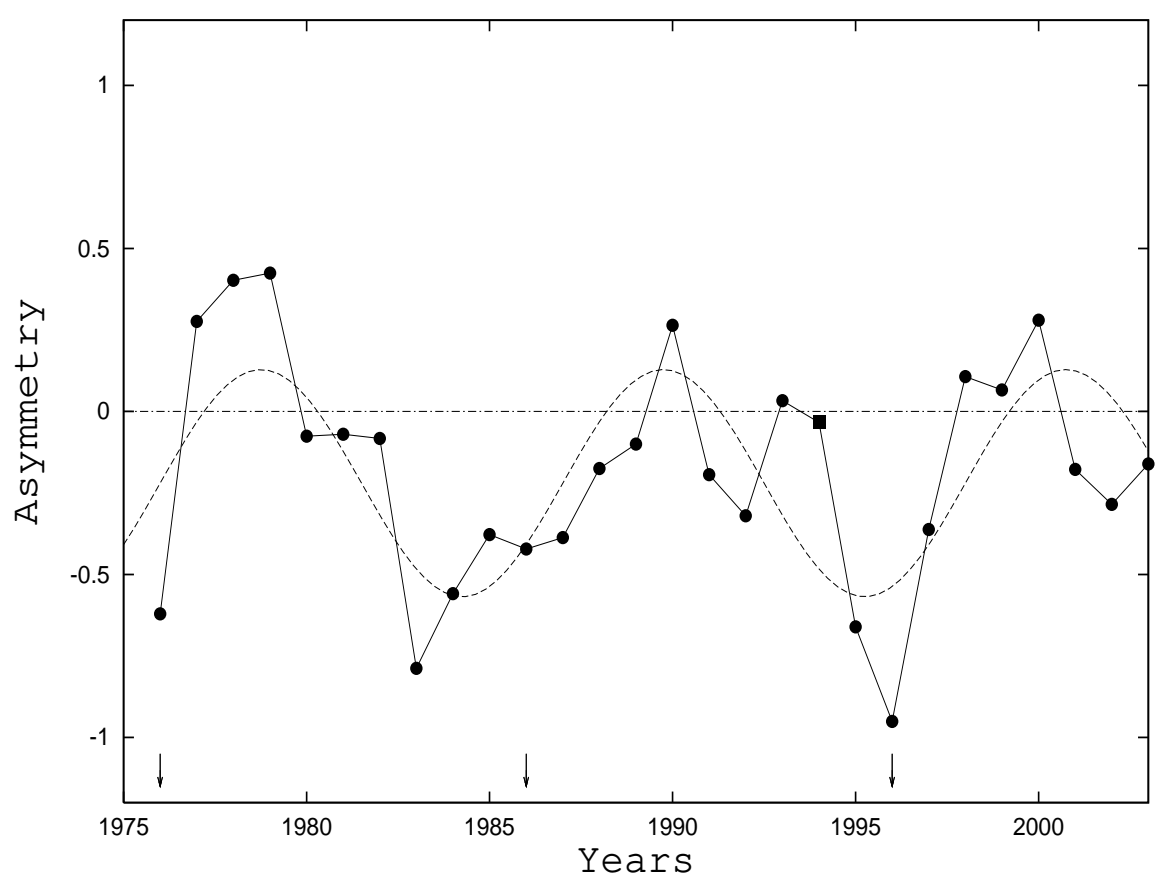

Figure 4. Yearly N-S asymmetry index of $F I_{S X R}$ during 1976-2003. Highly significant asymmetry values with $\mathrm{p} \geq 99.5 \%$ are marked with circles, otherwise squares are drawn. Dashed line corresponds to the fitted sinusoidal curve with a period of eleven years. Downward arrows indicate solar activity minima.

not due to random fluctuations. We have also studied the asymmetry of $F I_{S X R}$ year wise (see Figure 4). In this case we have found that out of 28 cases, the data is highly significant $(\mathrm{p}>99.5 \%)$ in 27 cases.

We are also interested to see any possible connection of the asymmetric behaviour of $F I_{S X R}$ with a period of eleven years. For this, in Figure 4, a comparison between the original asymmetry curve and a fitted sinusoidal function is also presented. The fitted sinusoidal function is of the form

$$
y(x)=C+A \sin 2 \pi f\left(t-t_{0}\right)
$$

where $C$ is a constant term and $A$ is the amplitude. $t_{0}$ has been assigned a value 1976. The frequency $f$ has been given a fixed value corresponding to eleven year period. Thus $f=1 / 11$ or 0.091 years ${ }^{-1}$. The values of $C$ and $A$ come out to be -0.22 and 0.35 respectively. We find that there is a good agreement between original curve of asymmetry index and the fitted sinusoidal curve. 


\section{Power Spectra of Asymmetry Time Series}

Using the Lomb-Scargle periodogram method (Lomb, 1976; Scargle, 1982; Horne and Baliunas, 1986) for the daily asymmetry time series, which is plotted in Figure 3, we have performed power spectra analysis. Figures 5a, 6a and 7a show the normalized power spectra of asymmetry time series during 1975-2003 for the three frequency ranges of 116.0-463.0, 16.5-116.0 and $7.7-16.5 \mathrm{nHz}$ which corresponds to the period intervals of $100-25,700-100$ and $1500-700$ days respectively. The $F I_{S X R}$ is not independent. Therefore the probability $\mathrm{P}$ of the power density at a given frequency being greater than $\mathrm{K}$ by chance is given by

$$
P(z>K)=\exp (-K / k),
$$

where the normalization factor $k$, which is due to event correlation, can be determined empirically (Bai and Cliver, 1990).

Figures $5 \mathrm{~b}, 6 \mathrm{~b}$ and $7 \mathrm{~b}$ show the distribution of the power values corresponding to the normalized power spectra shown in Figures 5a, $6 \mathrm{a}$ and $7 \mathrm{a}$ respectively. The vertical axis shows the cumulative number of frequencies for which the power exceeds a certain value. For example, in the power spectrum for $116.0-463.0 \mathrm{nHz}$ range shown in Figure $5 \mathrm{a}$ we have 1222 frequencies. For all these frequencies the power exceeds zero; thus, we have a point at $(\mathrm{X}=0, \mathrm{Y}=1222)$. At only one frequency (409.5 nHz, which is equivalent to 28.26 days) the power was 30.02 , its maximum value. For lower values of power, the distribution can be well fitted by the equation $\mathrm{y}=1226 \exp (-\mathrm{x} / 3.7)$, as expected from equation (6). Thus, we normalize the power spectrum by dividing the powers by 3.7 to obtain Figure 5a. For other cases we use the same procedure for normalization.

Once the power spectrum has been normalized properly, we can use the 'False alarm probability' (FAP) formula to estimate the statistical significance of a peak in the power spectrum. It is given by the expression

$$
F=1-\left[1-\exp \left(-Z_{m}\right)\right]^{N},
$$

where $Z_{m}$ is the height of the peak in the normalized power spectrum and $\mathrm{N}$ is the number of independent frequencies. Therefore, for example, in the case of power spectrum shown in Figure 5a, if we substitute $Z_{m}=8.13$ and $\mathrm{N}=1222$ (since we searched 1222 frequencies with 0.284 $\mathrm{nHz}$ intervals) in equation ( 7 ) we get $\mathrm{F}=0.30$, i.e., the probability to obtain such a high peak at $409.5 \mathrm{nHz}$ (28.26 days) by chance is about $30 \%$. The same analysis has been applied to the power spectra in other two frequency ranges. In the frequency interval of $16.5-116.0 \mathrm{nHz}$ and 

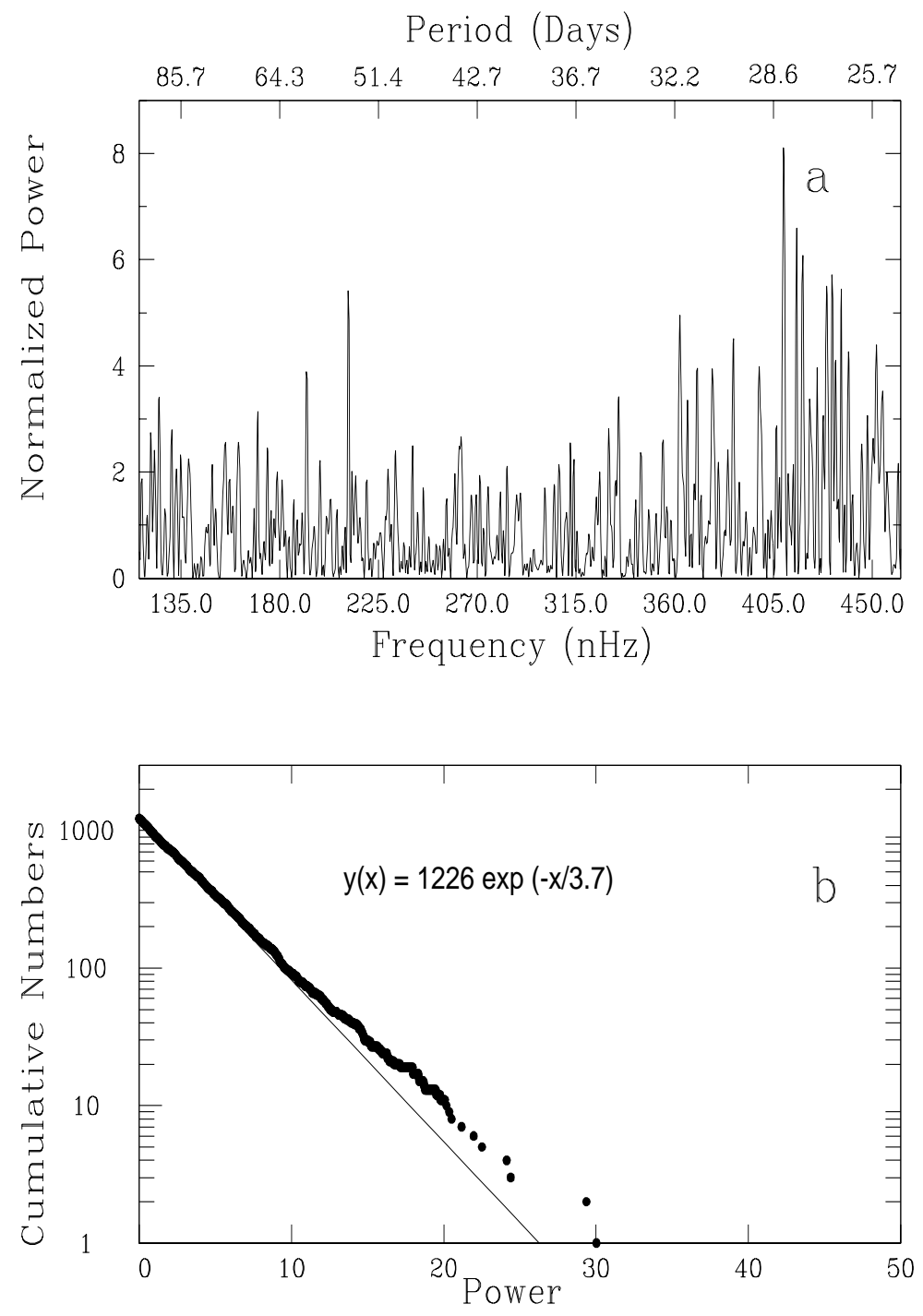

Figure 5. (a) Normalized power spectrum of the daily asymmetry time series for the frequency interval of $116.0-463.0 \mathrm{nHz}$ (100-25 days). (b) Scargle power distribution corresponding to Figure 5a. The vertical axis is the number of frequencies for which power exceeds $\mathrm{x}$. The straight line is the fit to the points for lower values of power. 

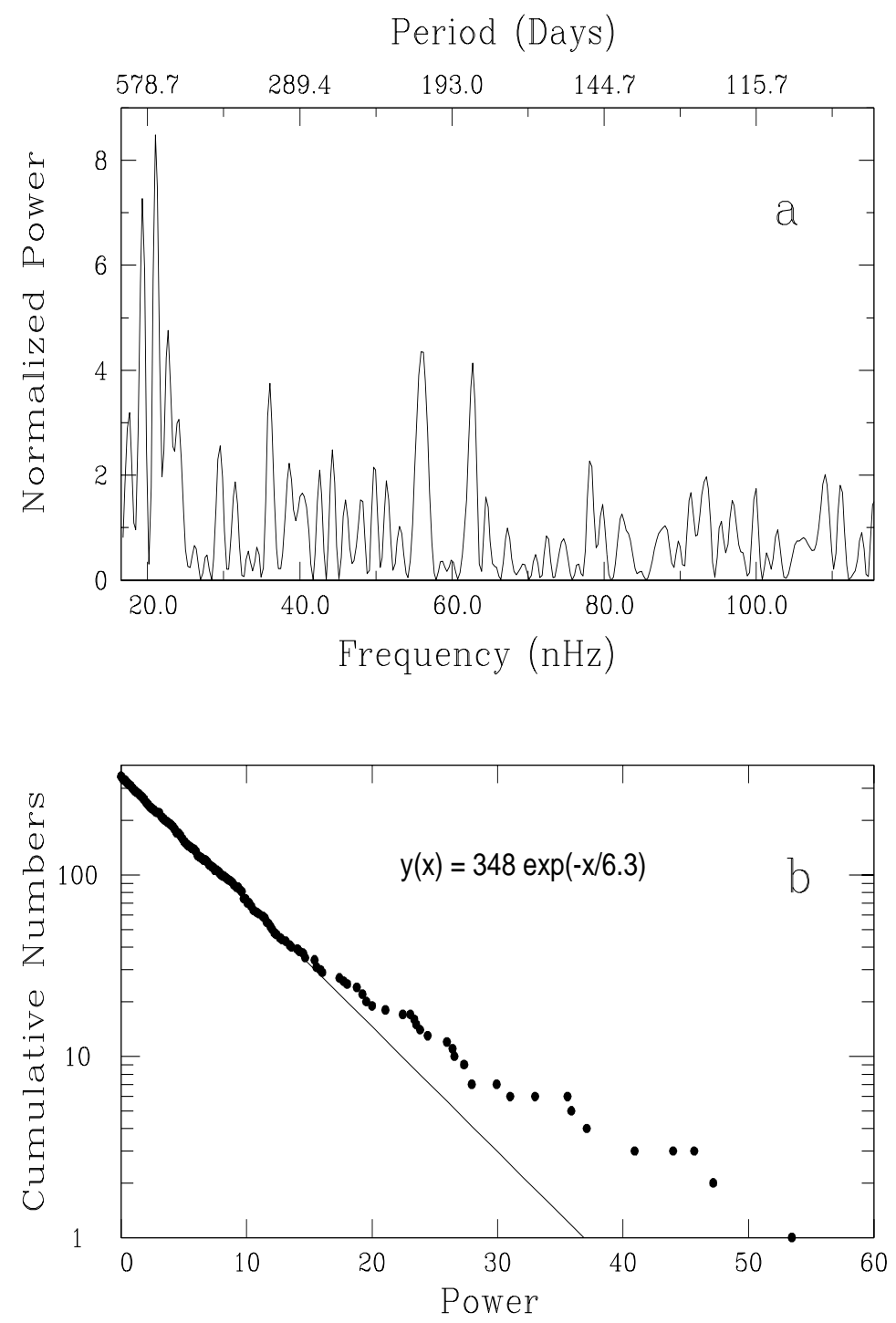

Figure 6. Same as Figure 5, but for the frequency interval of $16.5-116.0 \mathrm{nHz}$ (700-100 days).

$7.7-16.5 \mathrm{nHz}$ we get important peaks at $21.02 \mathrm{nHz}(550.73$ days $)$ with a $\mathrm{FAP}=7 \%$ and $8.5 \mathrm{nHz}$ (3.72 years) with a $\mathrm{FAP}=20.4 \%$ respectively.

To confirm the peaks of 28.26 days, 550.73 days and 3.72 years in Figures 5a, 6a and 7a respectively are not due to aliasing, we have removed sine curves of these periods from the original time series. Then, once again, we have computed the periodogram (see Figure 8). 

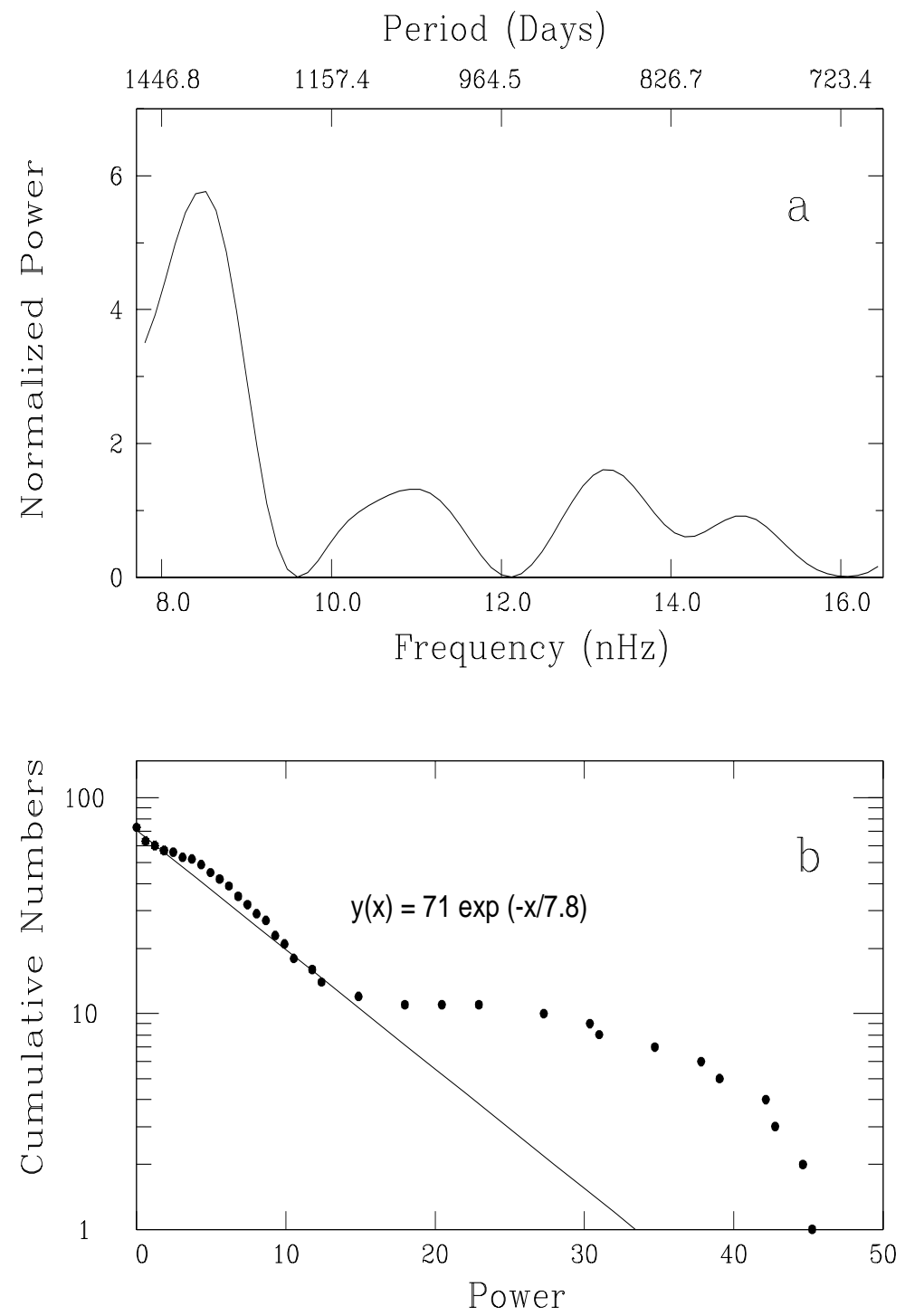

Figure 7. Same as Figure 5, but for the frequency interval of $7.7-16.5 \mathrm{nHz}$ (1500-700 days).

\section{Discussions and Conclusions}

In this paper we have calculated the daily $F I_{S X R}$ for solar cycles 21 to 23. The plot (Figure 1) of monthly values of $F I_{S X R}$ indicates a lower level of activity during solar cycle 23 compared to previous two cycles. This has been pointed out earlier by Özgüç et al. (2002) in his study during the rising phase of solar cycle 23. The scatter diagram (Figure 

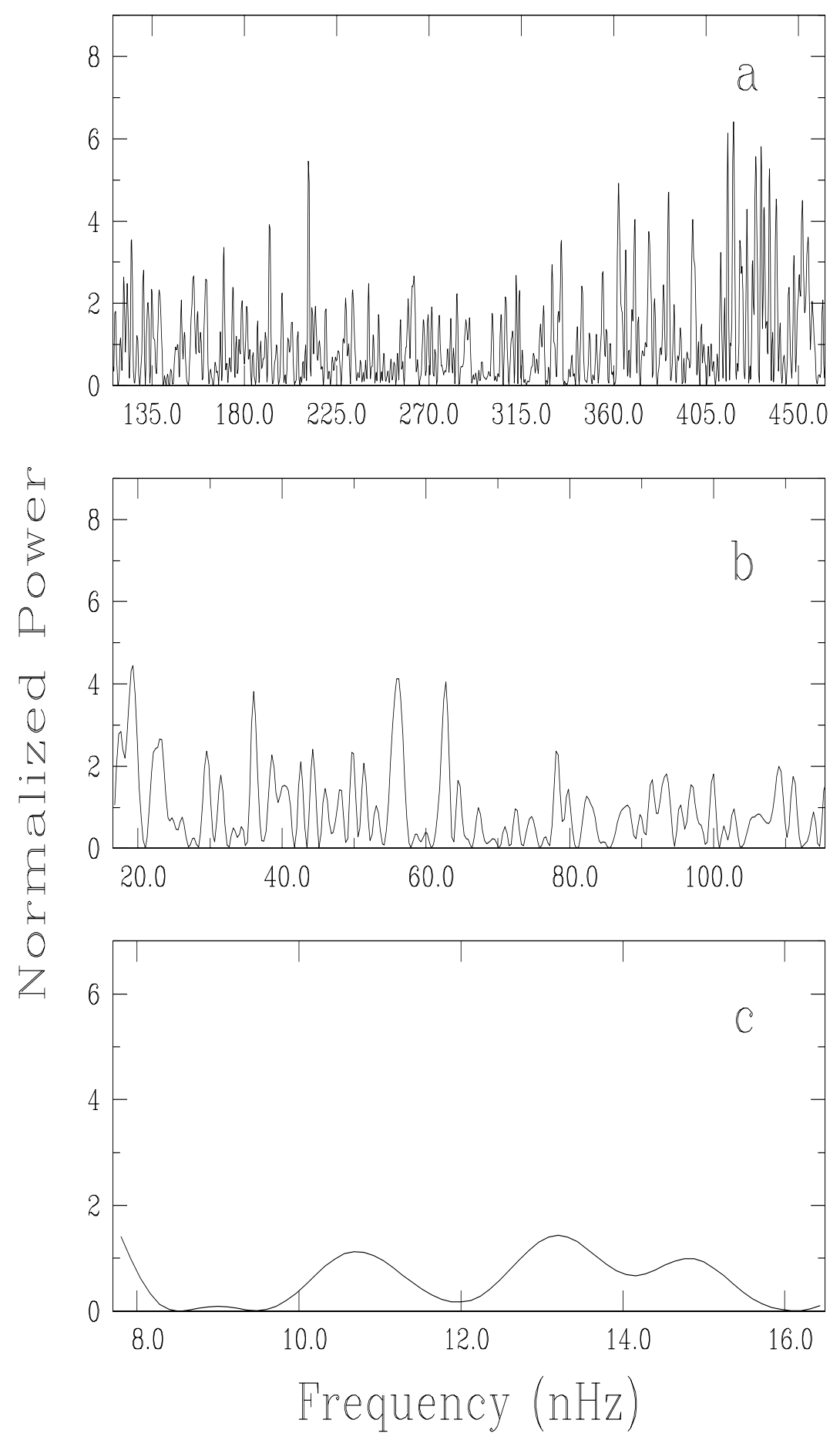

Figure 8. Periodogram of asymmetry time series, for the frequency intervals of (a) $116.0-463.0 \mathrm{nHz}$ (b) $16.5-116.0 \mathrm{nHz}$ and (c) $7.7-16.5 \mathrm{nHz}$, after subtraction of a sine curve with frequency $409.5 \mathrm{nHz}$ (28.26 days), $21.02 \mathrm{nHz}$ (550.73 days) and 8.5 $\mathrm{nHz}$ (3.72 years) respectively. 
2) between $F I_{S X R}$ and $F I_{H \alpha}$ shows that there exists a good correlation between these two solar activity indices. Ataç and Özgüç (1989, 1998, 2003 ) in their various papers have made extensive statistical analysis of $F I_{H \alpha}$, finding its good correlation with a number of solar indices that arise under different physical conditions. Therefore, a good correlation of $F I_{S X R}$ with $F I_{H \alpha}$ also makes the former an interesting parameter which is good enough to describe the solar activity.

We have found that there exists a real $\mathrm{N}-\mathrm{S}$ asymmetry in $F I_{S X R}$ which is not due to random fluctuations. The fitted straight line to asymmetry time series (Figure 3 ) for the cycles 21 and 23 shows that the activity in the northern hemisphere is more important during the ascending branch of cycle whereas during the descending branch the activity becomes more important in the southern hemisphere. In the cycle 22 there was a southern dominance throughout. The slope of regression lines for all the three cycles is found to be negative. Vizoso and Ballester (1990), in his study of $\mathrm{N}-\mathrm{S}$ asymmetry of sunspot area during solar cycles 12-20 and sunspot number for cycle 21, found that the slope of regression lines fitted to the yearly values of $\mathrm{N}-\mathrm{S}$ asymmetry change its sign each four cycles suggesting a long term periodic behaviour in the N-S asymmetry of around eight cycles. They also found that the slope of regression lines has changed from positive to negative values after cycle 19. Ataç and Özgüç (1996) made similar conclusions with the N-S asymmetry of $F I_{H \alpha}$ during cycles 17-22. Our study confirms and extends these earlier results. Moreover, Ataç and Özgüç (1996) suggested that, if the period of eight cycles is real, the behaviour of asymmetry during cycles 20-22 should be maintained in cycle 23, which has also been confirmed by the present study.

On the other hand, if we consider the plot of yearly N-S asymmetry index (Figure 4) the change in the predominance of activity in the two hemisphere can be studied for every year starting from 1976. Initially during the solar cycle 21, the northern hemisphere was more active but after 1980 it was shifted slightly towards south. In the year 1983 the activity moved towards south strongly and prevailed there during most of the solar cycle 22 . In the solar cycle 23 , we observe a strong peak in southern hemisphere during the minimum phase in the year 1996 and then the activity is moved towards the north. In the years 1998, 1999 and 2000 the northern hemisphere was dominated. These results are in agreement with the work done by Temmer et al. (2001), who analyzed the N-S asymmetry of $H \alpha$ flares from 1975 to 1999. The preference for northern hemisphere during the rising phase of cycle 23 is also reported by Ataç and Özgüç (2001) with the data of $F I_{H \alpha}$. Here we also notice that, during cycle 23 , the behaviour of asymmetry is changed in the year 2000 and it has dominated towards southern hemisphere in the 
successive years. The Figure also reveals that the fitted sinusoidal curve with a period of eleven years reflects the behaviour of asymmetry in a good manner. This suggests that $\mathrm{N}-\mathrm{S}$ asymmetry of $F I_{S X R}$ has a periodic behaviour shifted in phase with respect to solar cycle. The shape of the curve also indicates that the asymmetry has peaked at or around the minimum of solar activity. This result is complementary to the study of $\mathrm{N}-\mathrm{S}$ asymmetry made by several authors (Swinson, Koyama and Saito, 1986; Vizoso and Ballester, 1990; Joshi, 1995; Ataç and Özgüç, 1996).

Finally, we have studied periodicities in the daily asymmetry time series for three frequency ranges. In the power spectrum for the frequency range of $116.0-463.0 \mathrm{nHz}$ (Figure 5), we get a significant peak at 409.5 $\mathrm{nHz}$ (28.26 days). It is close to the 27 days solar rotational period which is well known in different solar indices. Antonucci et al. (1990) investigated the rotation of photospheric magnetic field during solar cycle 21 , and obtained a dominant period of 26.9 days for the northern and 28.1 days for the southern hemisphere. A similar outcome is obtained by Temmer et al. (2002) from the power spectral analysis of daily sunspot numbers during 1975-2000 in which they derive a rigid rotation with 27.0 days for the northern hemisphere, while the southern hemisphere reveals a dominant period of 28.2 days. Pap, Tobiska, and Bouwer (1990) and Bouwer (1992) reported periods between 26-28 days from the power spectra of various solar indices. Joshi (1999) reported peaks at 26.5 days and 28.3 days in the power spectrum of solar radio flux at $10.7 \mathrm{~cm}$. Lou et al. (2003) found a prominent peak of around 28 days in the daily averaged Ap index for geomagnetic disturbances, which is physically identified with solar rotation. The power spectrum for the $16.5-116.0 \mathrm{nHz}$ range (Figure 6) shows a significant peak at $21.02 \mathrm{nHz}$ (550.73 days). Ichimoto et al. (1985) reported that a peak in the power spectra appears near 510-540 days when northern and southern hemisphere flares are analyzed separately. Bai (1987) found an important peak at 552 days in the power spectra of major flares belonging to northern hemisphere. A significant peak of around 20 rotations (about 540 days) is found by Akioka et al. (1987) in the power spectra for TA (Sum of maximum areas of sunspot groups per solar rotation) and MA (mean area of sunspot groups per solar rotations) during solar cycle 21. Özgüç and Ataç (1989) reported a peak at 564 days in the $F I_{H \alpha}$ during cycle 20 and 21 considered together. Oliver, Carbonell, and Ballester (1992) found the periodicities around 540 days in the cycles 12, 14 and 17 in sunspot areas, while during cycles 18 and 19 it is present, with a very high significance, in sunspot and Zurich sunspot number. They also found a peak at 528 days in the periodogram for sunspot areas during cycles 12 to 21 . In the power spectra for the frequency range 
of $7.7-16.5 \mathrm{nHz}$ (Figure 7), we have a significant peak at $8.5 \mathrm{nHz}$ (3.72 years). Rao (1973) studied periodicities in several indices of solar activity and found that the index $\mathrm{P}$ (average area of all spot groups), $T_{0}$ (average spot group life time) and $f_{0}$ (number of all spot groups formed all over the Sun within a unit time) presented periodicities around 3.9, 3.5 and 3.48 years, respectively. Vizoso and Ballester (1989) found a periodicity of around 3.1-3.2 years in the values of $F I_{H \alpha}$ and flare numbers N-S asymmetry. A periodicity of 3.27 years is reported by Vizoso and Ballester (1990) in the N-S asymmetry of sunspot areas. In all the three power spectra presented by us, the subtraction of sine curve indicates that the peaks are probably real and not spurious or due to aliasing (Figure 8). We, therefore, see that the periodicities in the asymmetry time series of $F I_{S X R}$ is consistent with the earlier results.

\section{Acknowledgements}

We are thankful to Prof. Ram Sagar for valuable comments and suggestions. One of the authors (BJ) wishes to thank Mr. Brijesh Kumar and Mr. J. C. Pandey for help in data analysis.

\section{References}

Akioka, M., Kubota, J., Suzuki, M., Ichimoto, K., and Tohmura, I.: 1987, Solar Phys. 112, 313.

Antalová, A.: 1996, Contrib. Astron. Obs. Skalnaté Pleso. 26, 98.

Antonucci, E., Hoeksema, J. T., and Scherrer, P. H. : 1990, Astrophys. J. 360, 296.

Ataç, T. and Özgüç, A.: 1996, Solar Phys. 166, 201.

Ataç, T. and Özgüç, A.: 1998, Solar Phys. 180, 397.

Ataç, T. and Özgüç, A.: 2001, Solar Phys. 198, 399.

Bai, T.: 1987, Astrophys. J. 318, L85.

Bai, T. and Cliver, E. W..: 1990, Astrophys. J. 363, 299.

Bai T. and Sturrock, P. A.: 1991, Nature 352, 360.

Bell, B.: 1962, Smithsonian Contr. Astrophys. 5, 203.

Bouwer, S. D.: 1992, Solar Phys. 142, 365.

Carbonell, M., Oliver, R., and Ballester, J. L.: 1993, Astron. Astrophys. 274, 497.

Horne, J. H. and Baliunas, S. L.: 1986, Astrophys. J. 302, 757.

Ichimoto, K., Kubota, J., Suzuki, M., Tohmura, I., and Kurokawa, H.: 1985, Nature

316, 422 .

Joshi, A.: 1995, Solar Phys. 157, 315.

Joshi, A.: 1999, Solar Phys. 185, 397.

Kleczek, J.: 1952, Publ. Contr. Astron. No. 22, Prague.

Kleczek, J.: 1953, Publ. Astrophys. Obs. Czech. Acad. Sci. No. 24, Prague.

Knoška, Š.: 1985, Contrib. Astron. Obs. Skalnaté Pleso 13, 217. 
Landi, R., Moreno, G., Storini, M., and Antalová, A.: 1998, J. Geophys. Res. 103, No. A9, 20553.

Letfus, V.: 1960, Bull. Astron. Inst. Czech. 11, 31.

Li, K. J., Schmieder, B., and Li, Q. S.: 1998, Astron. Astrophys. 131, 99.

Li, K. J., Wang, J.X., Xiong, S. Y., Liang, H. F., Yun, H. S., and Gu, X. M.: 2002, Astron. Astrophys. 383, 648.

Lomb, N.: 1976, Astrophys. Space Sci. 39, 477.

Lou, Y., Wang, Y., Fan, Z., Wang, S., and Wang, J.: 2003, Monthly Notices Royal Astron. Soc. , In press.

Oliver, R., Carbonell, M., and Ballester, J.L.: 1992, Solar Phys. 137, 141.

Oliver, R., Ballester, J.L.: 1994, Solar Phys. 152, 481.

Özgüç, A. and Ataç, T.: 1989, Solar Phys. 123, 357.

Özgüç, A. and Ataç, T.: 1996, Solar Phys. 163, 183.

Özgüç, A., Ataç, T., and Rybák, J.: 2002, J. Geophys. Res. 107, No. A7, 10.1029/2001JA009080.

Özgüç, A., Ataç, T., and Rybák, J.: 2003, Solar Phys. 214, 375.

Pap, J., Tobiska, W.K., and Bouwer, S. D.: 1990, Solar Phys. 129, 165.

Rao, R.: 1973, Solar Phys. 29, 47.

Roy, J. R.: 1977, Solar Phys. 52, 53.

Scargle. J. D.: 1982, Astrophys. J. 263, 835.

Swinson, D. B., Koyama, H, and Saito, T.: 1986, Solar Phys. 106, 35.

Temmer, M., Veronig, A., Hanslmeier, A., Otruba, W., and Messerotti, M..: 2001, Astron. Astrophys. 375, 1049.

Temmer, M., Veronig, A., and Hanslmeier, A..: 2002, Astron. Astrophys. 390, 707.

Verma, V. K.: 1993, Astrophys. J. 403, 797.

Vizoso, G. and Ballester, J. L.: 1987 Solar Phys. 112, 317.

Vizoso, G. and Ballester, J. L.: 1989 Solar Phys. 119, 411.

Vizoso, G. and Ballester, J. L.: 1990 Astron. Astrophys. 229, 540.

Address for Offprints: Bhuwan Joshi

State Observatory,

Manora Peak, Naini Tal-263 129,

INDIA.

Phone: +91-05942-235583, 235136

Fax: +91-05942-235136

email: bhuwan@upso.ernet.in 
paper.tex; 13/06/2021; 14:48; p.16 